\title{
Advancing Remote Sensing and Machine Learning-Driven Frameworks for Groundwater Withdrawal Estimation in Arizona: Linking Land Subsidence to Groundwater Withdrawals
}

\author{
Sayantan Majumdar ${ }^{1}$, Ryan Smith ${ }^{1}$, Brian Conway ${ }^{2}$, and V Lakshmi ${ }^{3}$ \\ ${ }^{1}$ Missouri University of Science and Technology \\ ${ }^{2}$ Arizona Department of Water Resources \\ ${ }^{3}$ University of Virginia
}

October 15, 2021

\begin{abstract}
Groundwater plays a crucial role in sustaining global food security but is being over-exploited in many basins of the world. Despite its importance and finite availability, local-scale monitoring of groundwater withdrawals required for sustainable water management practices is not carried out in most countries, including the United States. In this study, we combine publicly available datasets into a machine learning framework for estimating groundwater withdrawals over the state of Arizona. Here we include evapotranspiration, precipitation, crop coefficients, land use, well density, and watershed stress metrics for our predictions. We employ random forests to predict groundwater withdrawals from 2002-2020 at a $2 \mathrm{~km}$ spatial resolution using in-situ groundwater withdrawal data available for Arizona Active Management Areas (AMA) and Irrigation Non-Expansion Areas (INA) from 2002-2009 for training and 2010-2020 for validating the model respectively. The results show high training ( $\left.\mathrm{R}^{2}[?] \quad 0.86\right)$ and good testing $\left(\mathrm{R}^{2}[?] \quad 0.69\right)$ scores with normalized mean absolute error (NMAE) [?] 0.64 and normalized root mean square error (NRMSE) [?] 2.36 for the AMA/INA region. Using this method, we spatially extrapolate the existing groundwater withdrawal estimates to the entire state and observe the co-occurrence of both groundwater withdrawals and land subsidence in South-Central and Southern Arizona. Our model predicts groundwater withdrawals in regions where production wells are present on agricultural lands and subsidence is observed from Interferometric Synthetic Aperture Radar (InSAR), but withdrawals are not monitored. By performing a comparative analysis over these regions using the predicted groundwater withdrawals and InSAR-based land subsidence estimates, we observe a varying degree of subsidence for similar volumes of withdrawals in different basins. The performance of our model on validation datasets and its favorable comparison with independent water use proxies such as InSAR demonstrate the effectiveness and extensibility of our combined remote sensing and machine learning-based approach.
\end{abstract}

\section{Hosted file}

Majumdar_Manuscript_HP.docx available at https://authorea.com/users/441247/articles/541781advancing-remote-sensing-and-machine-learning-driven-frameworks-for-groundwaterwithdrawal-estimation-in-arizona-linking-land-subsidence-to-groundwater-withdrawals 


\begin{tabular}{l|c|c|c}
\hline \multirow{2}{*}{\multicolumn{1}{c|}{ AMA/INA }} & \multicolumn{3}{|c}{ Test Error Metric } \\
\cline { 2 - 4 } & $\boldsymbol{R}^{\mathbf{2}}$ & NRMSE & NMAE \\
\hline Phoenix (PHX) & 0.69 & 2.05 & 0.64 \\
Pinal (PIN) & 0.76 & 1.85 & 0.51 \\
Tucson (TUC) & 0.51 & 4.07 & 0.99 \\
Santa Cruz (SCA) & 0.66 & 2.79 & 0.75 \\
Prescott (PRE) & 0.59 & 3.24 & 1.01 \\
Harquahala (HAR) & 0.32 & 4.39 & 1.01 \\
Douglas (DIN) & 0.59 & 1.82 & 0.67 \\
Joseph City (JCI) & 0.76 & 2.29 & 0.77 \\
\hline
\end{tabular}

\begin{tabular}{|c|c|c|c|c|}
\hline $\begin{array}{l}\text { GW } \\
\text { Basin }\end{array}$ & $\begin{array}{l}\text { Mean } \\
\text { Sediment } \\
\text { Thickness }\end{array}$ & TS/TPGW & $\begin{array}{l}\text { Confinement of } \\
\text { principal aquifer }\end{array}$ & Reference \\
\hline RAN & 197.77 & 0.0001 & Unconfined & $\begin{array}{l}\text { Tillman, Cordova, Leake, } \\
\text { Thomas, and Callegary (2011) }\end{array}$ \\
\hline MMU & 325.96 & 0.0981 & Confined & Stolley et al. (2020) \\
\hline HAR & 361.45 & 0.0329 & $\begin{array}{l}\text { Unconfined/partially } \\
\text { confined }\end{array}$ & Stolley et al. (2020) \\
\hline PHX & 290.66 & 0.0038 & Unconfined & $\begin{array}{l}\text { M. M. Miller and Shirzaei } \\
(2015)\end{array}$ \\
\hline PIN & 339.97 & 0.0118 & Unconfined & Rascona (2006) \\
\hline TUC & 260.53 & 0.001 & Unconfined & Eastoe and $\mathrm{Gu}(2016)$ \\
\hline DOU & 12.07 & 0.0038 & Unconfined $^{\dagger}$ & Coates and Cushman (1955) \\
\hline DIN & 324.58 & 0.0947 & Partially confined & Coates and Cushman (1955) \\
\hline WIL & 240.79 & 0.1069 & Partially confined & Nelson et al. (2018) \\
\hline SAF & 207.95 & 0.0637 & Partially confined & Corkhill (2015) \\
\hline
\end{tabular}

$\dagger$ Wells along the edge of the basin, where this region lies, were interpreted by Coates and Cushman (1955) not to have confining layers.

${ }^{\ddagger}$ Most wells drilled in the area that is presently subsiding at the time Coates and Cushman (1955) were produced were shallow (average depth of $89 \mathrm{~m}$ ), while wells drilled after 1955 were much deeper (average depth of $145 \mathrm{~m}, A D W R, 2020)$. Coates and Cushman (1955) reported mostly unconfined conditions with confining conditions observed at many deep wells (100 $\mathrm{m}$ depth and greater). For this reason, we consider the principal aquifer at present to be partially confined. 

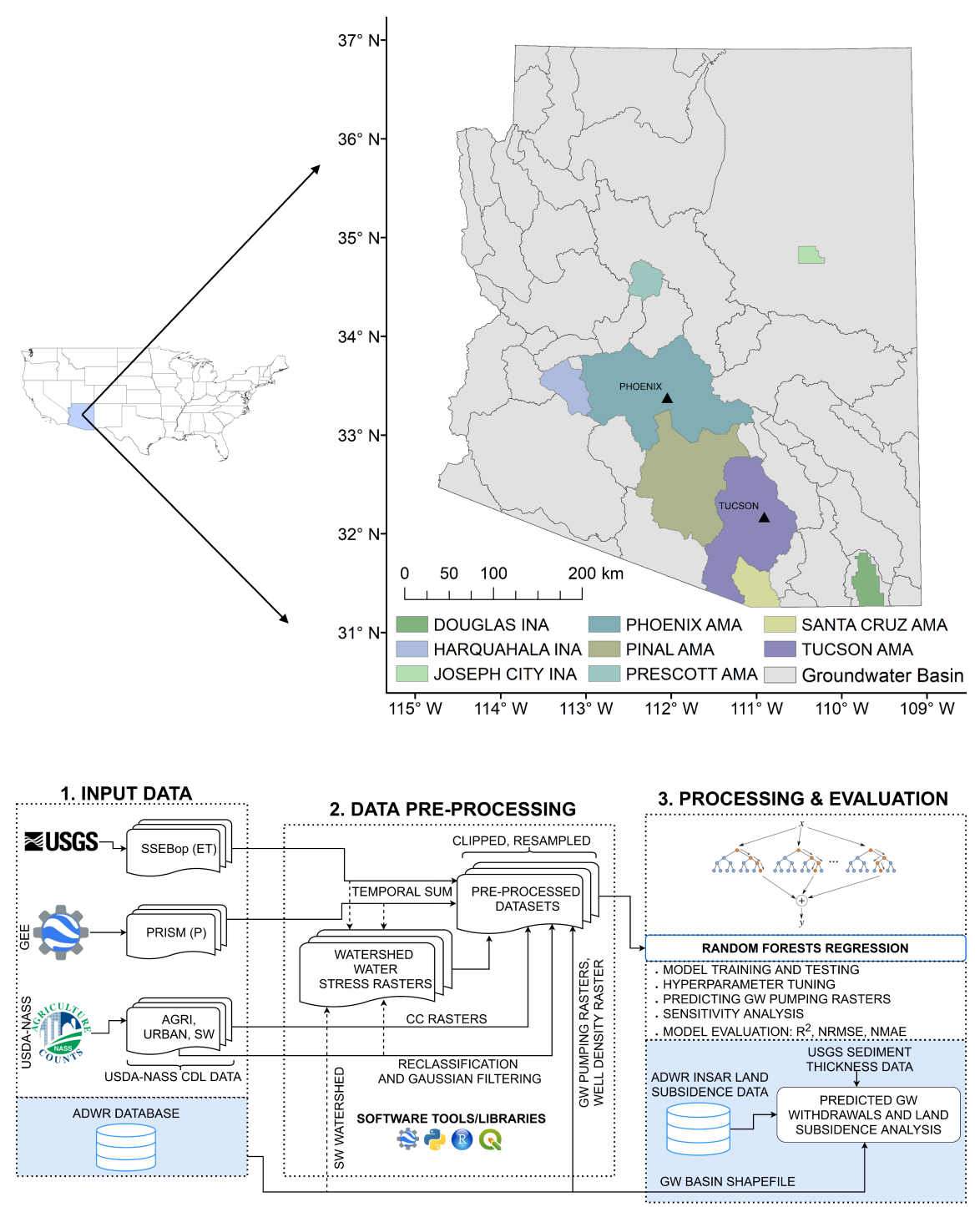


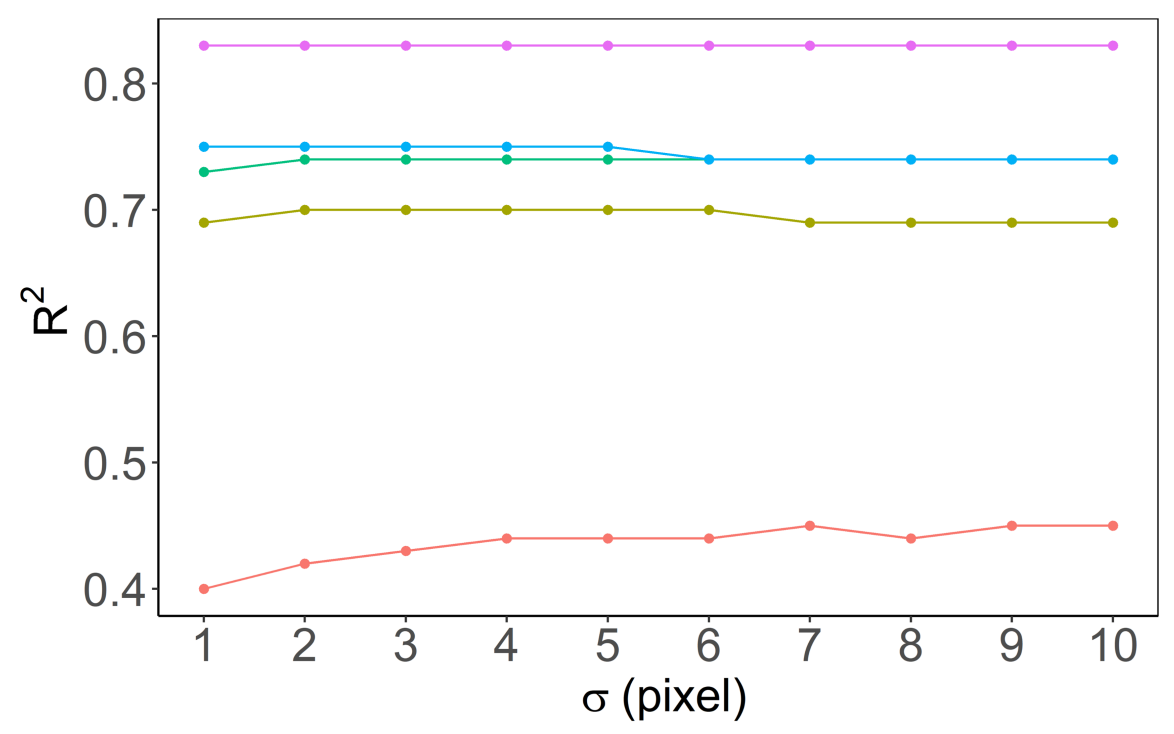

scale $(\mathrm{km}) \rightarrow 1 \rightarrow 2 \rightarrow 3 \rightarrow 4 \rightarrow 5$

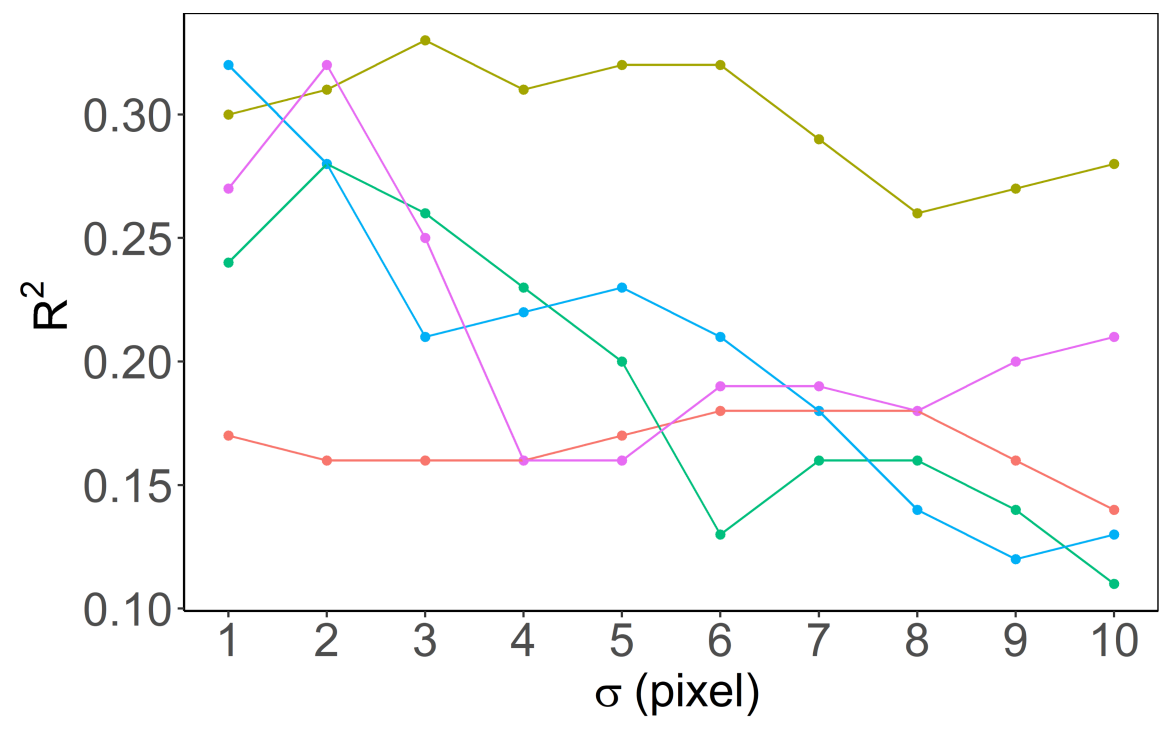

scale $(\mathrm{km}) \rightarrow 1 \rightarrow 2 \rightarrow 3 \rightarrow 4 \rightarrow 5$ 


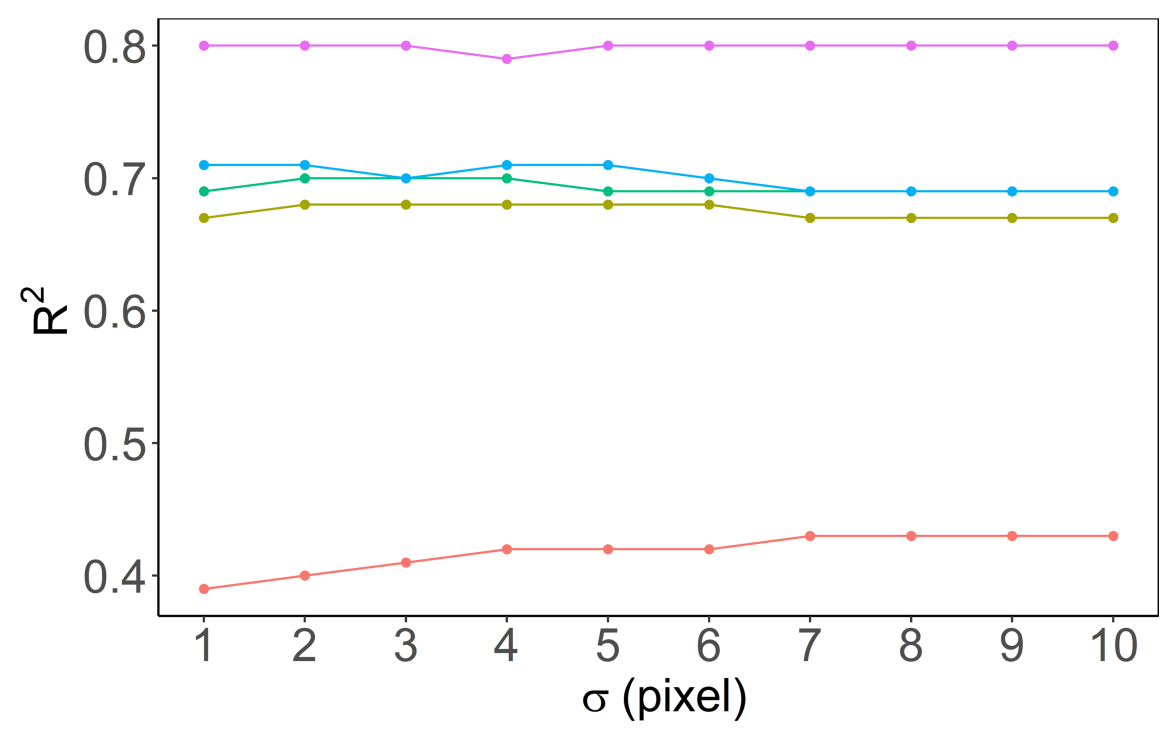

scale $(\mathrm{km}) \rightarrow 1 \rightarrow 2 \rightarrow 3 \rightarrow 4 \rightarrow 5$ 


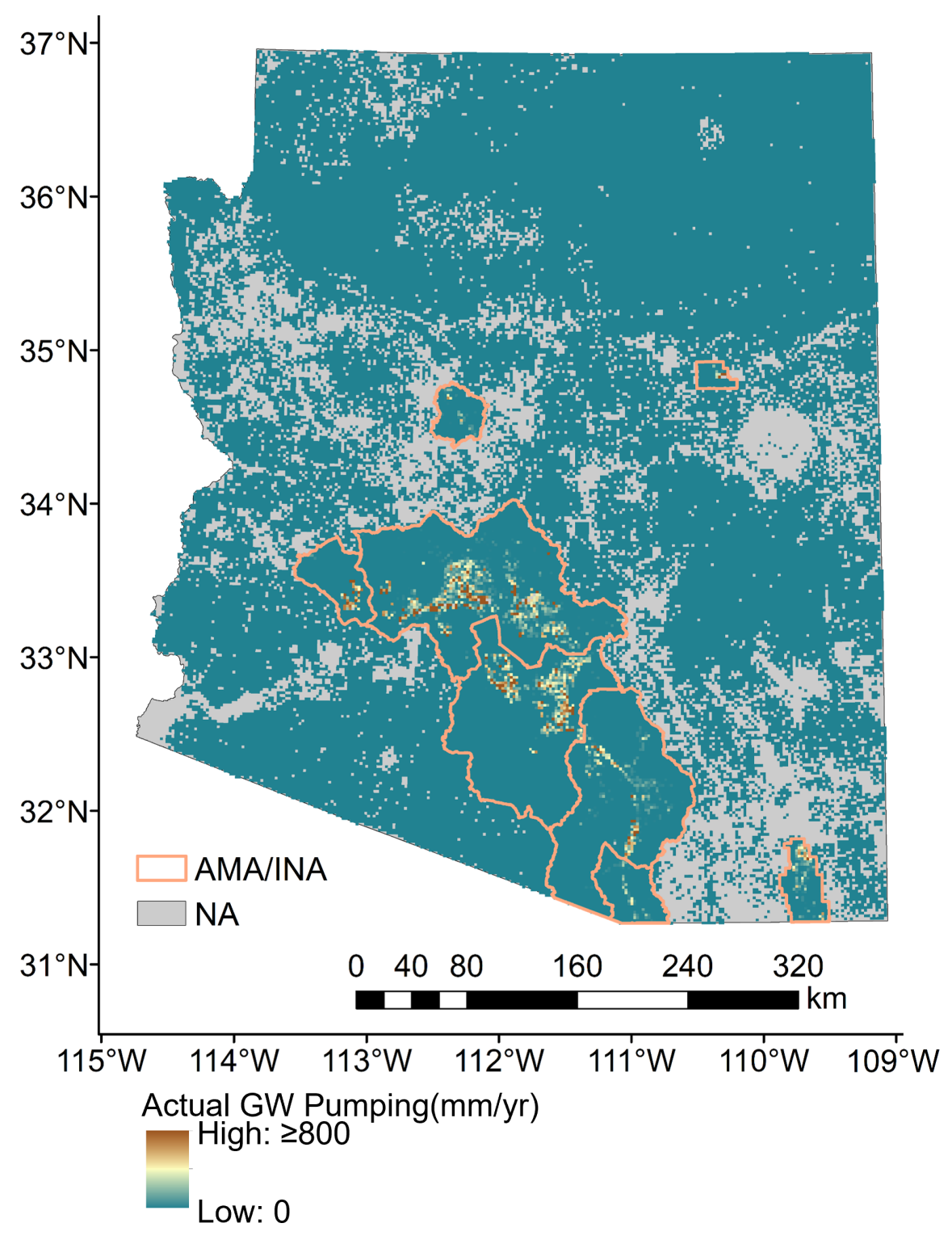




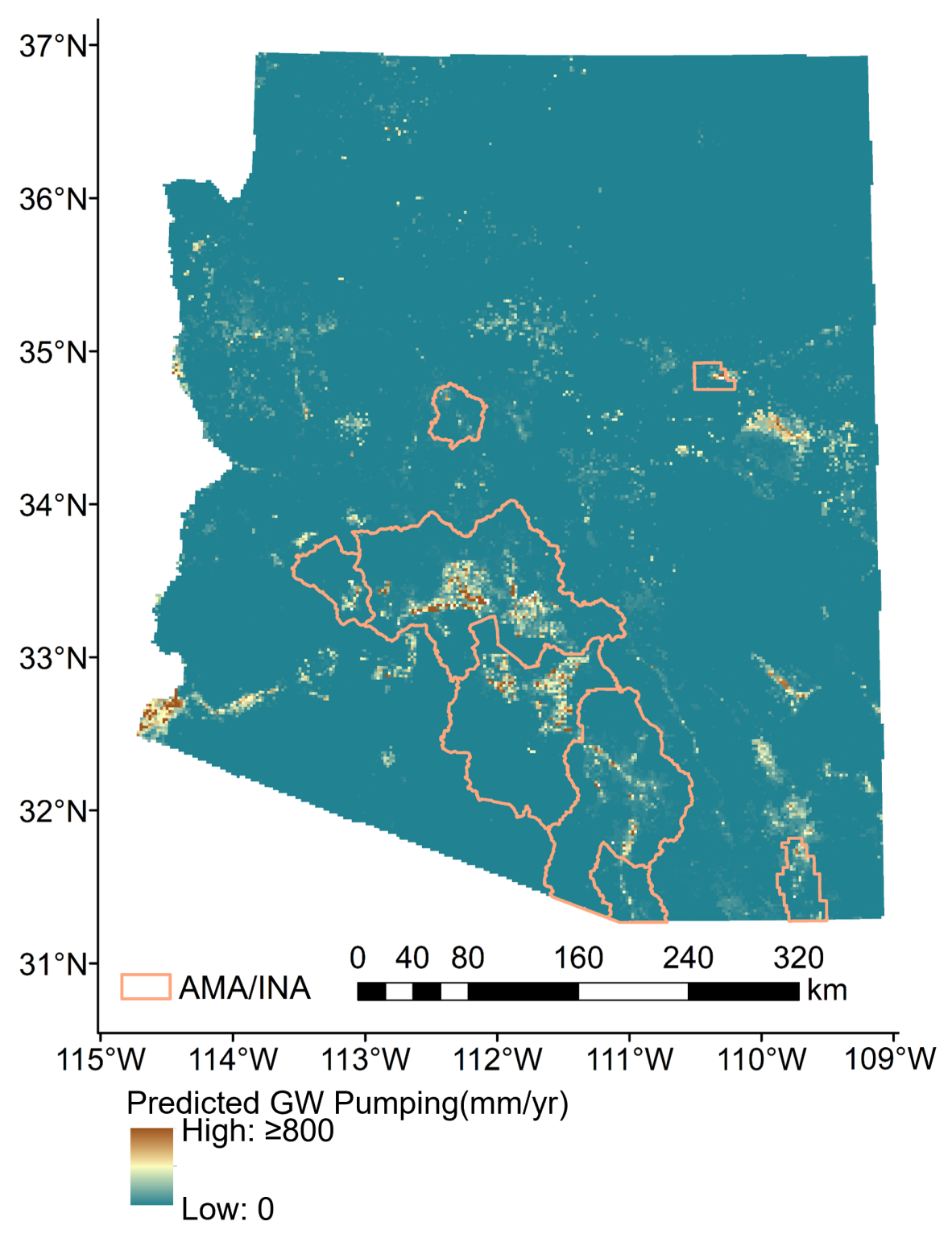



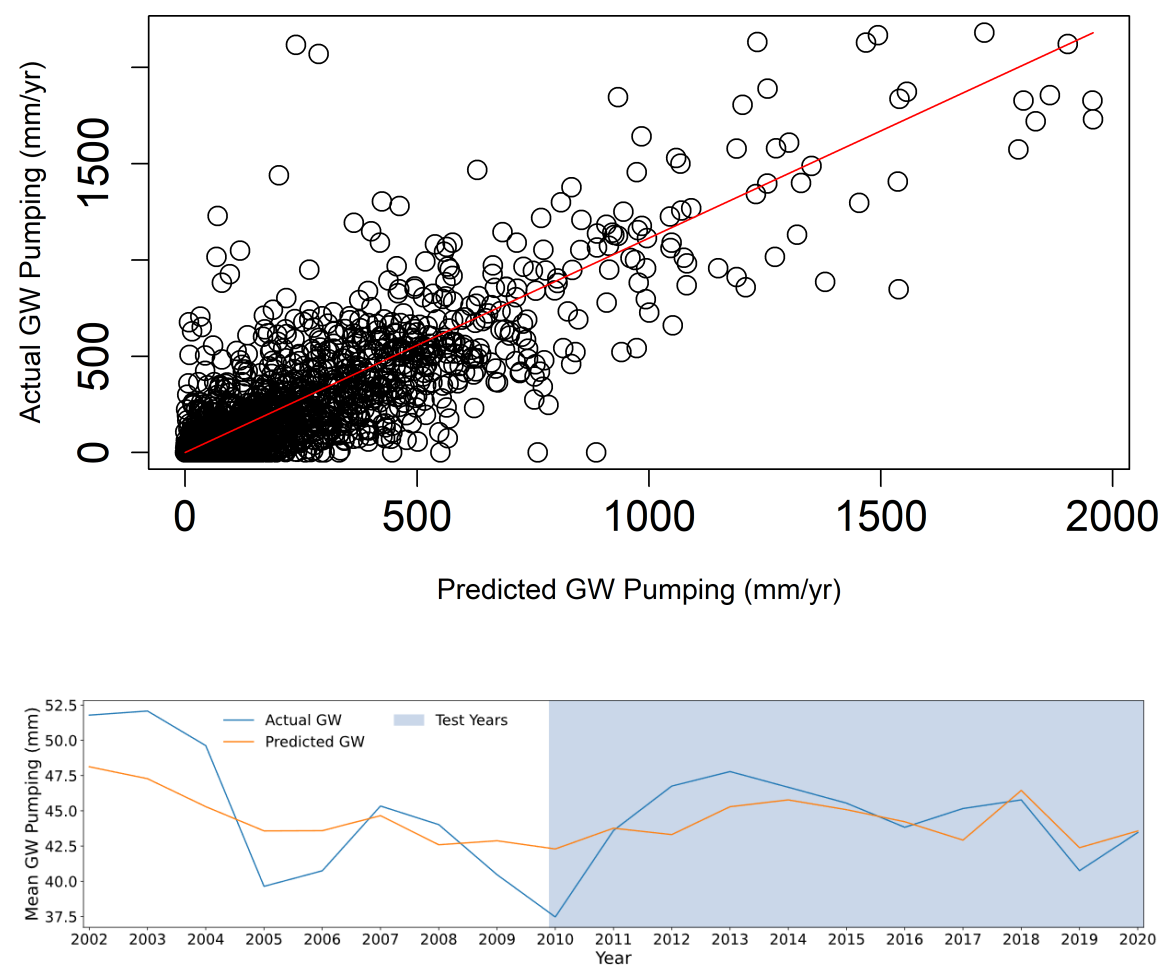


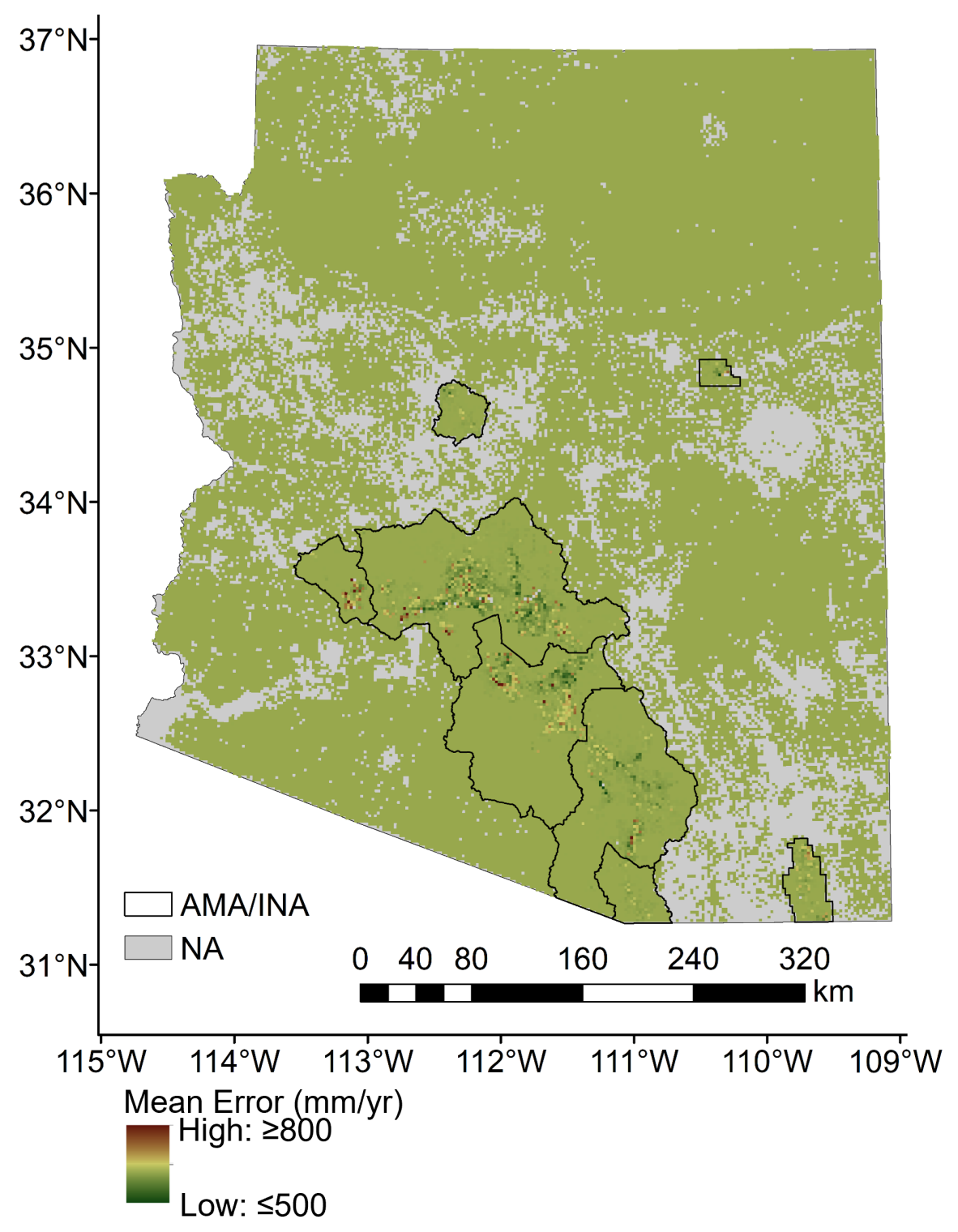



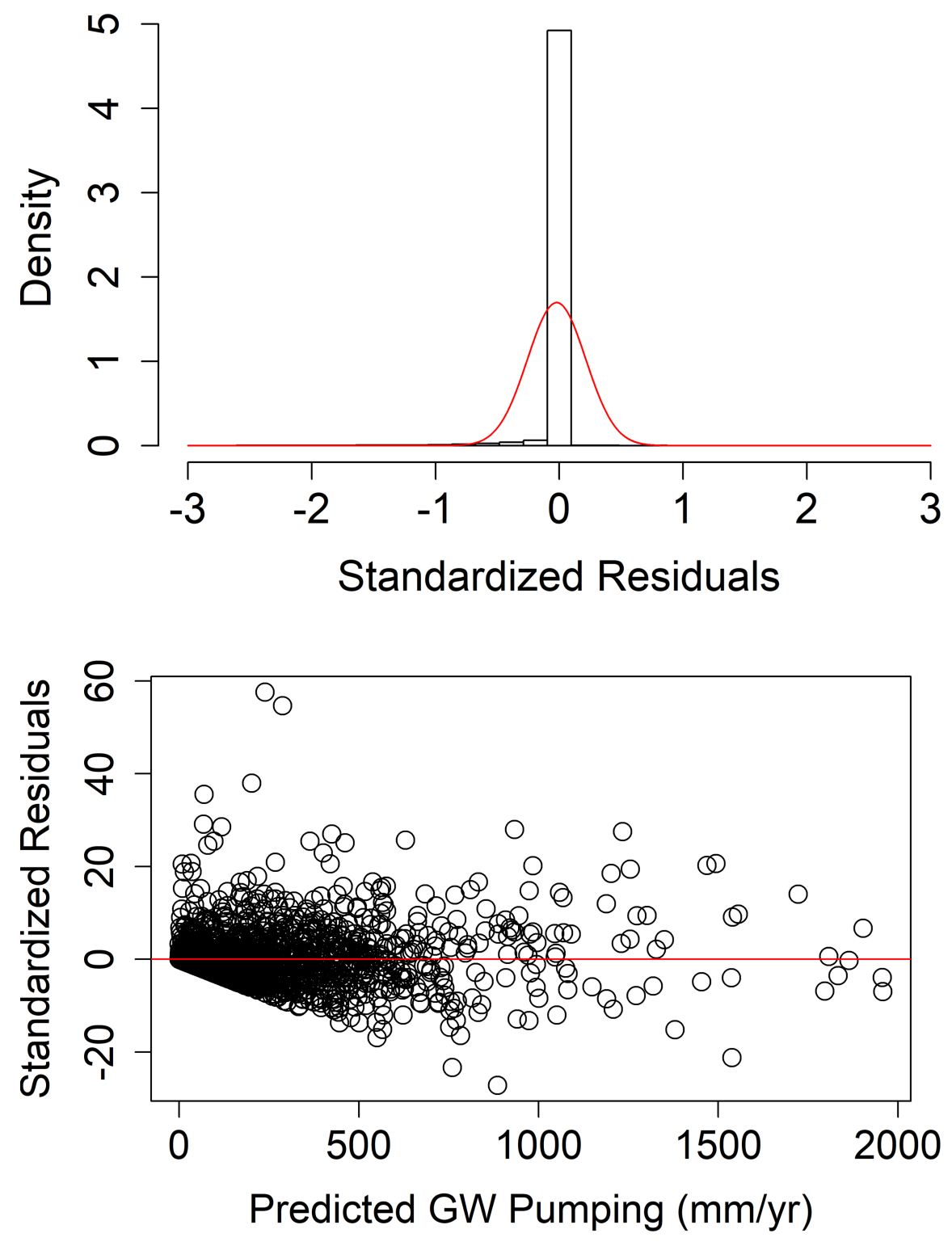

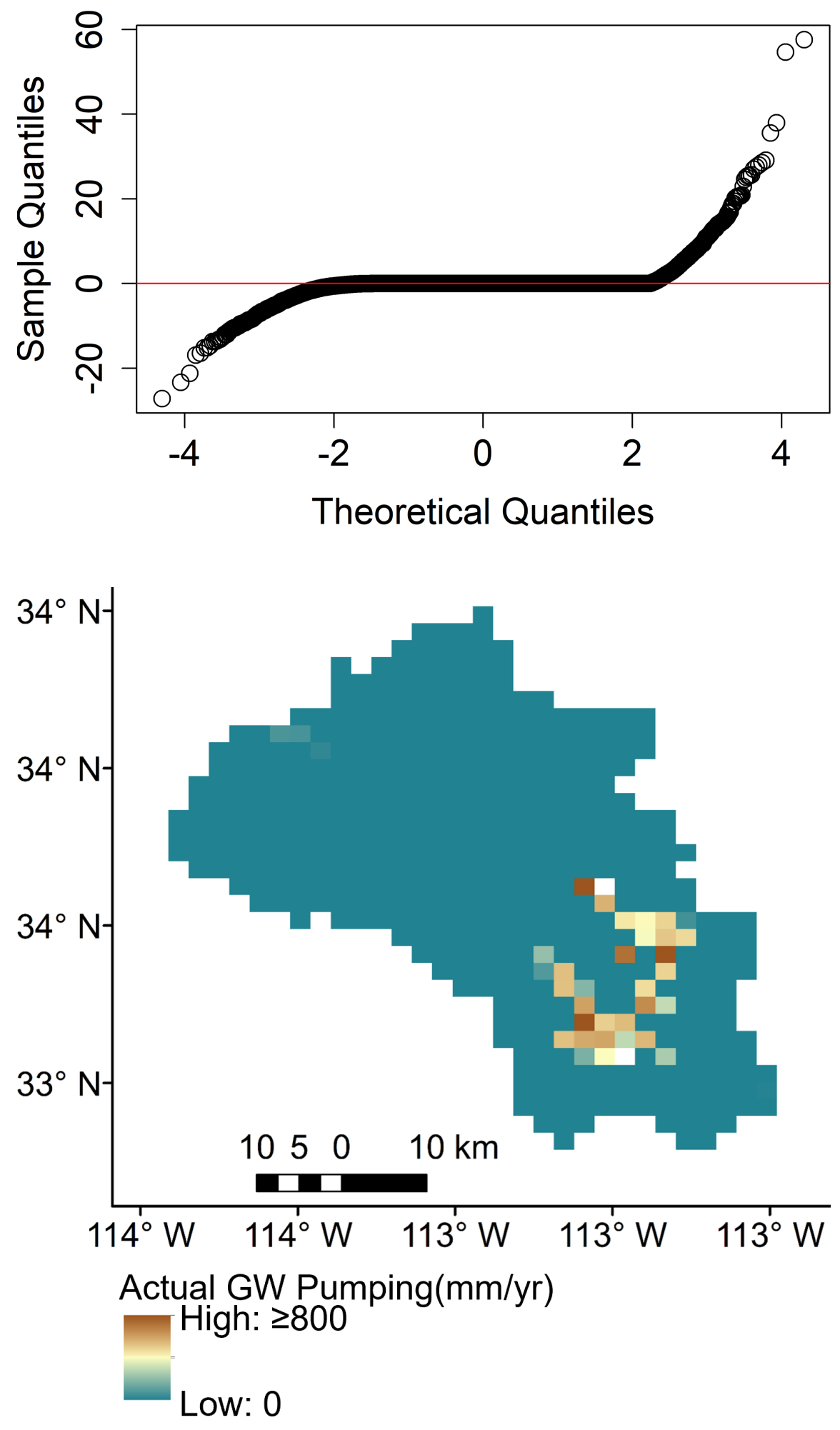

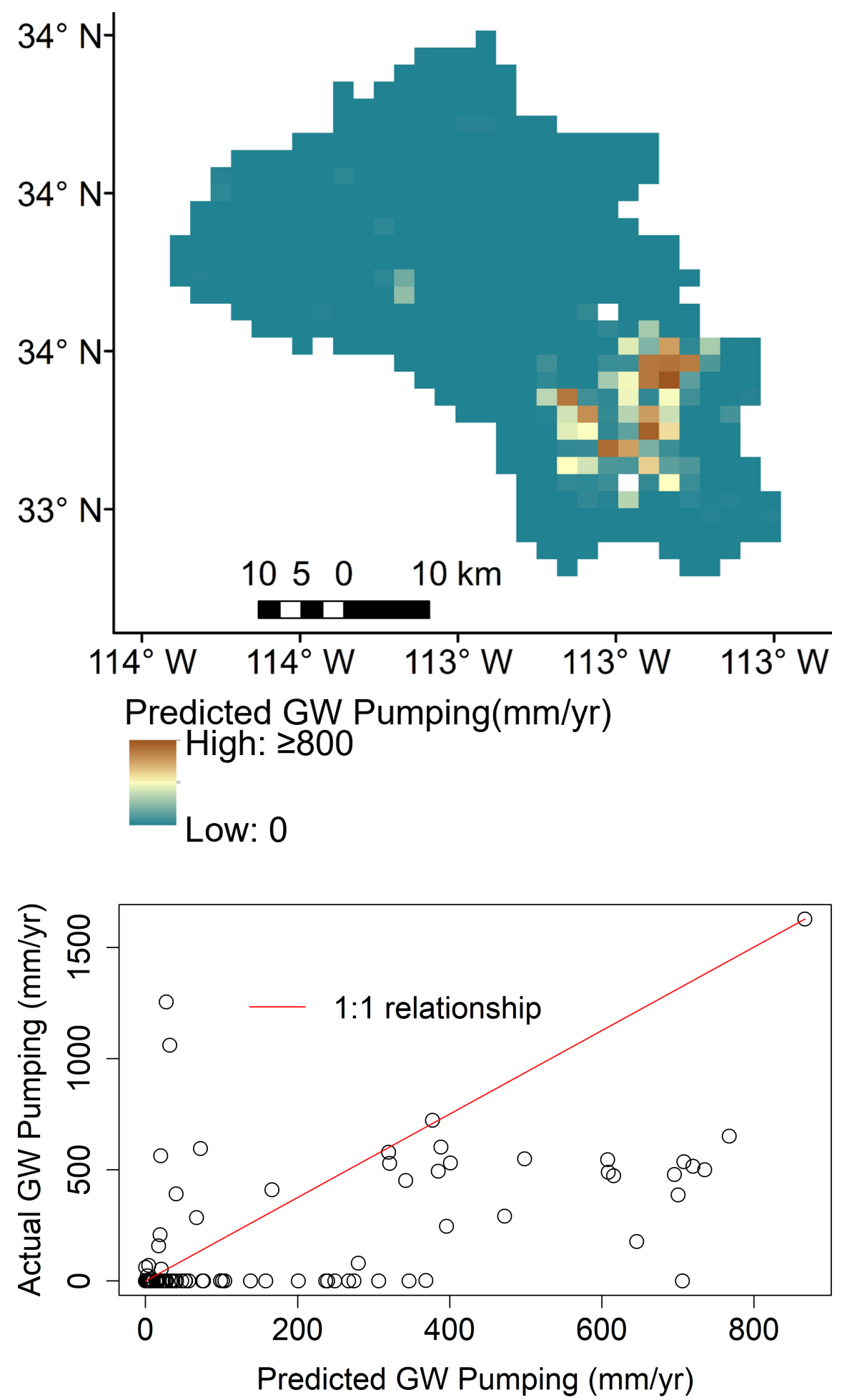

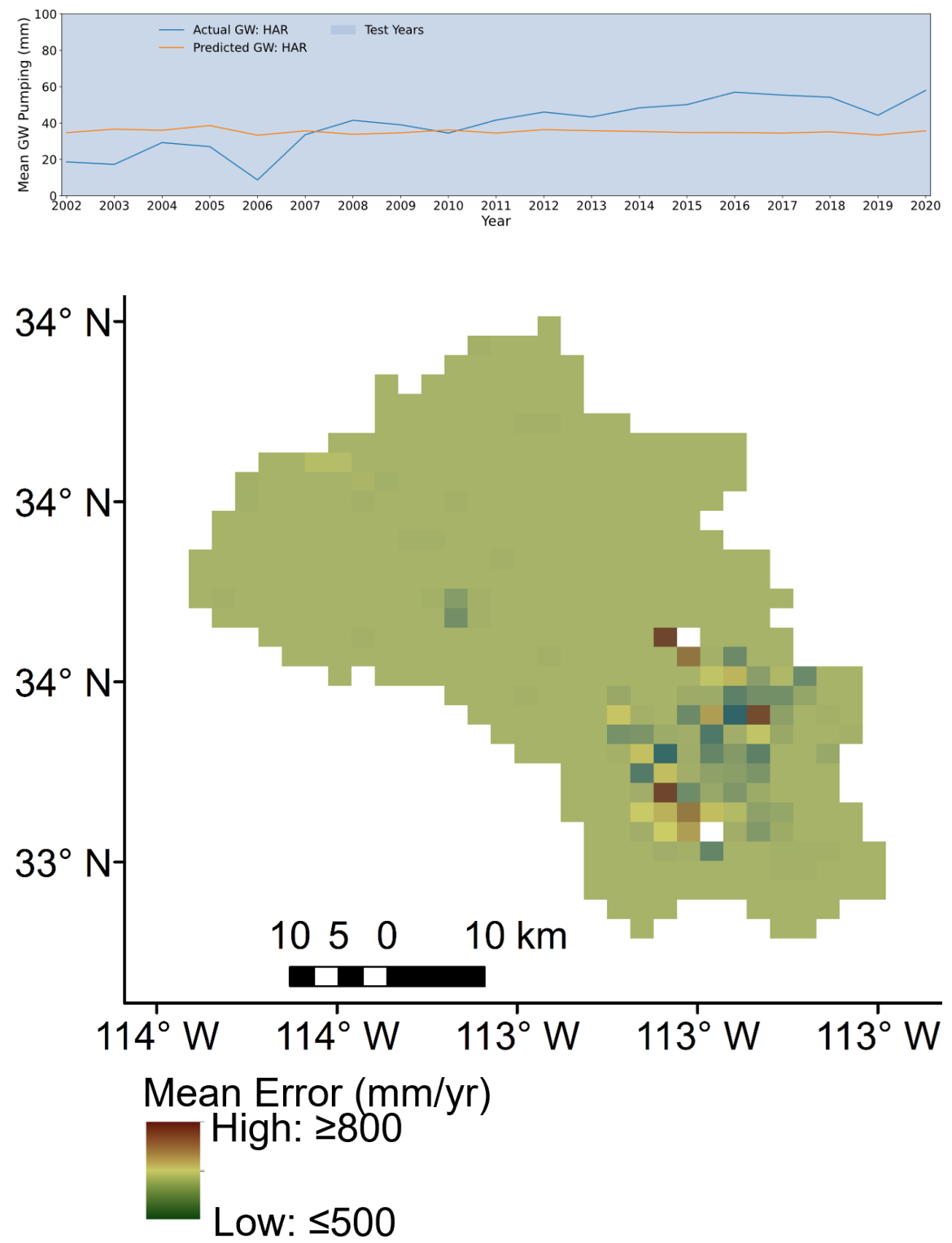

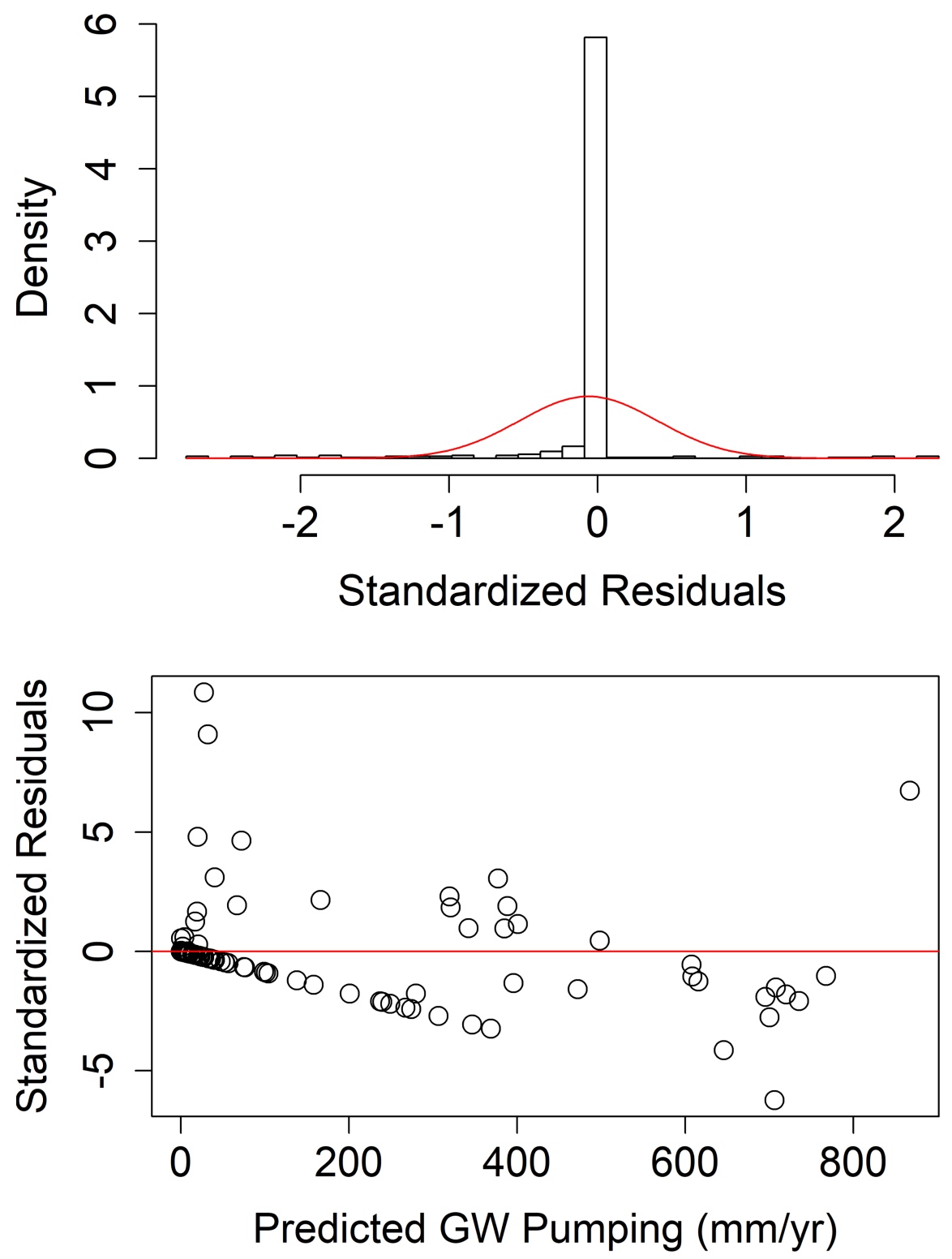

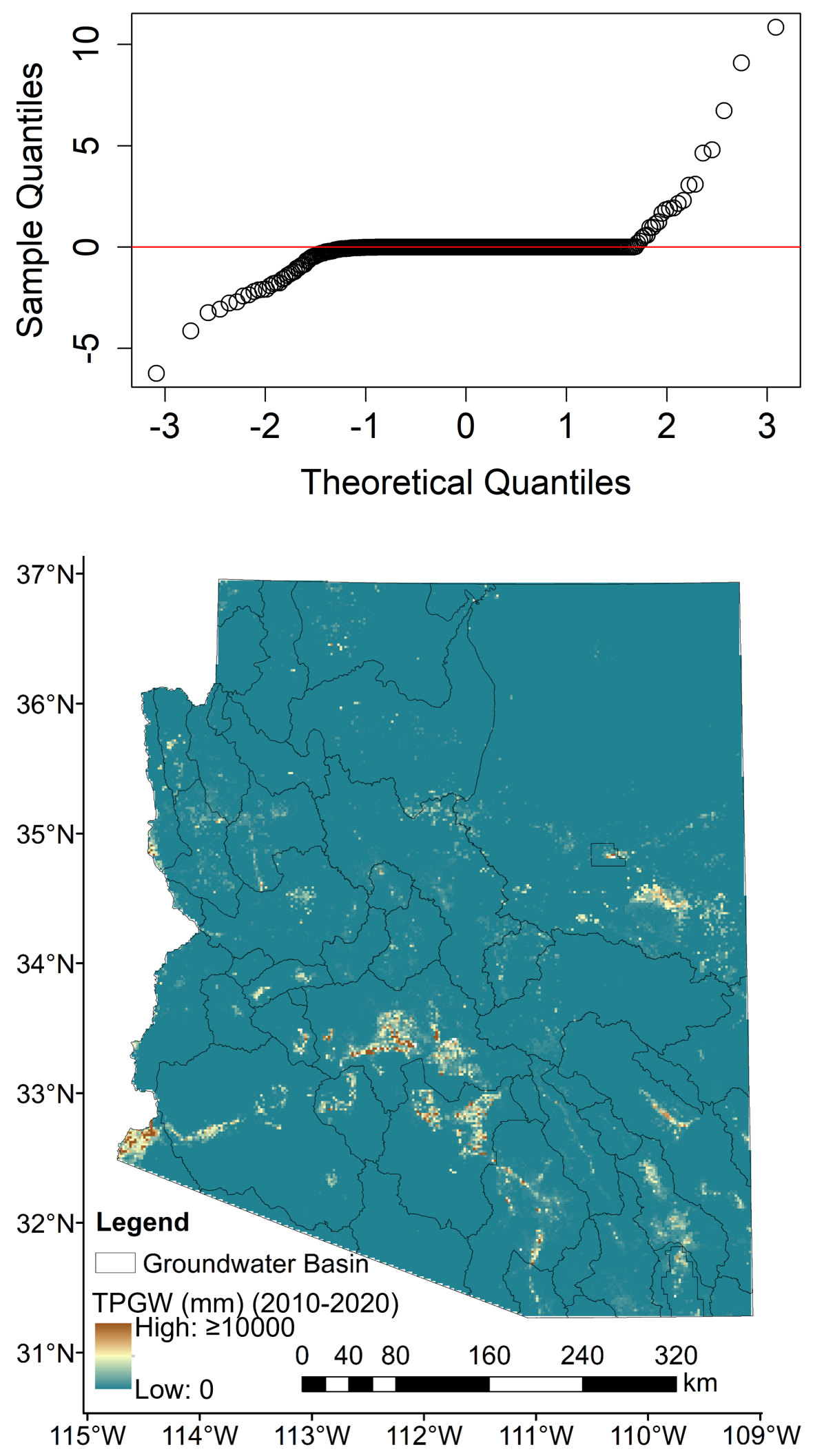


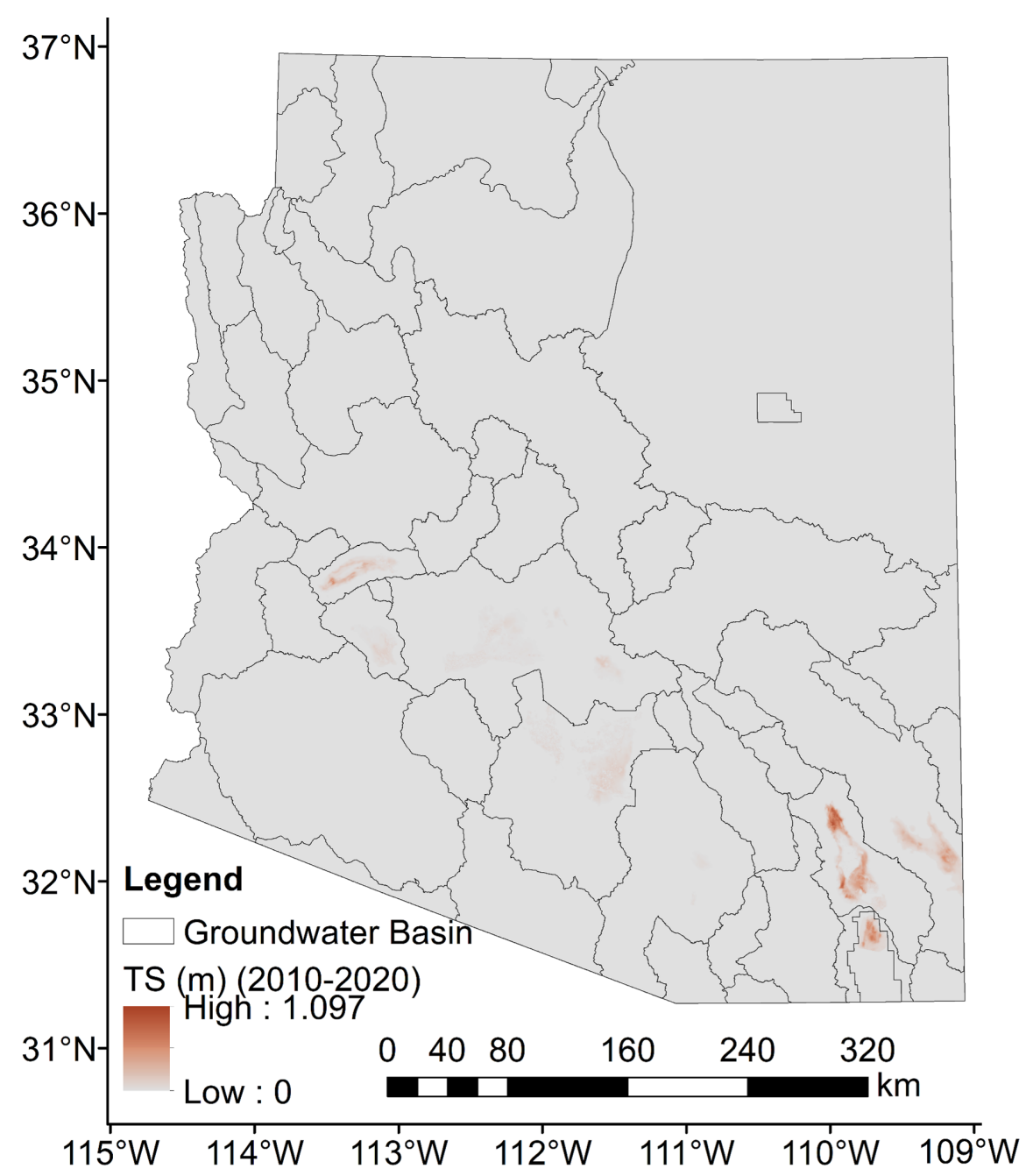




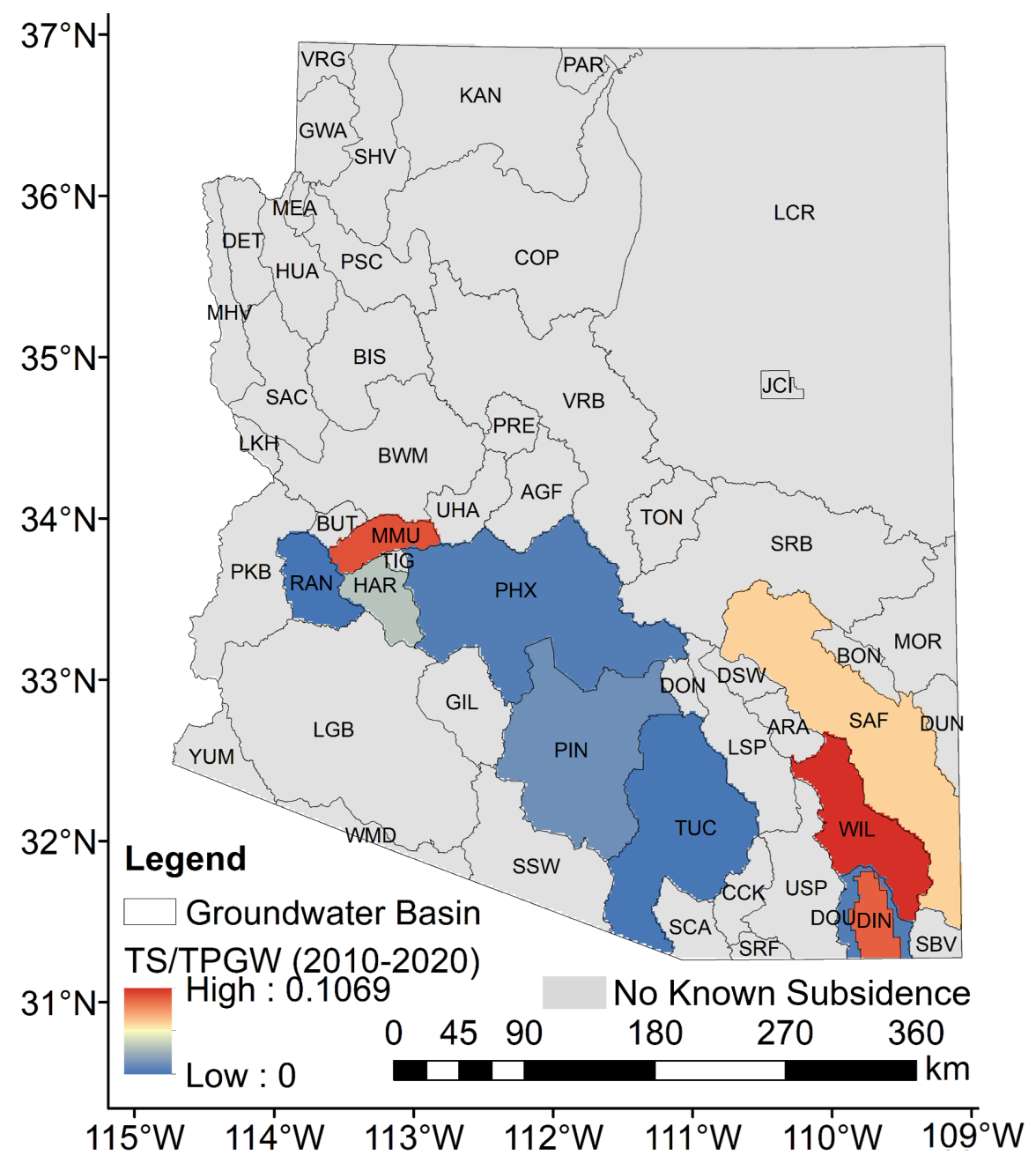




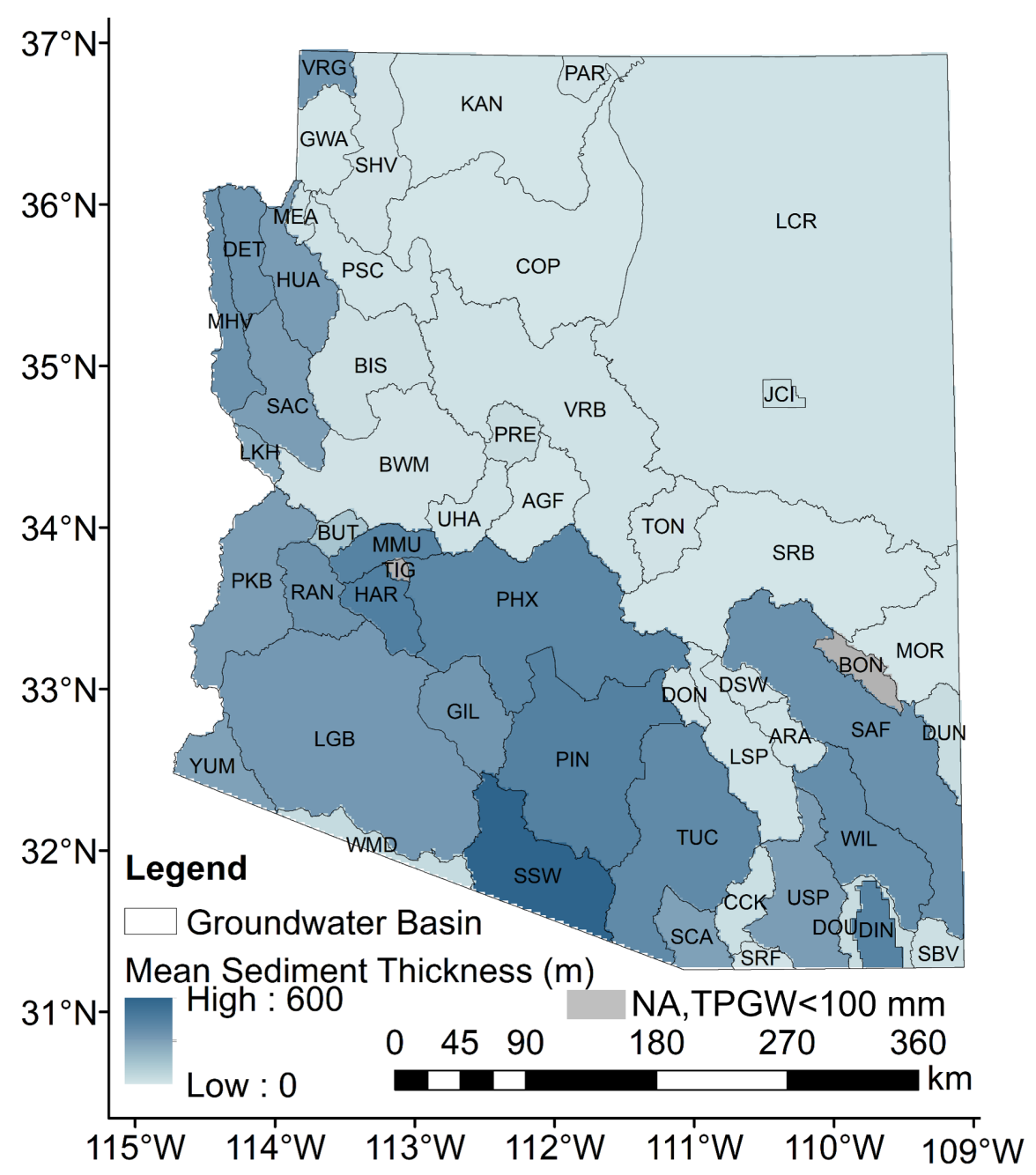

